\title{
The salience network dynamics in perceptual decision-making
}

Ganesh B. Chand ${ }^{1}$ and Mukesh Dhamala ${ }^{1,2,3, *}$

${ }^{1}$ Department of Physics and Astronomy, ${ }^{2}$ Neuroscience Institute, Georgia State University, Atlanta, GA, 30303, USA

${ }^{3}$ Center for Behavioral Neuroscience, Center for Nano-Optics, Center for Diagnostics and Therapeutics, GSU-GaTech Center for Advanced Brain Imaging, Georgia State University, Atlanta, GA, 30303, USA

*Correspondence:

Mukesh Dhamala, PhD

1 Park Place NE, Suite 433

Department of Physics and Astronomy, Georgia State University, Atlanta, Georgia, 30303, United States of America

Phone: 404-413-6043

Email:mdhamala@gsu.edu

Number of pages: 52

Number of figures: 8

Number of table: 1 


\section{Abstract}

Recent neuroimaging studies have demonstrated that the network consisting of the right anterior insula (rAI), left anterior insula (IAI) and dorsal anterior cingulate cortex (dACC) is activated in sensory stimulus-guided goal-directed behaviors. This network is often known as the salience network (SN). When and how a sensory signal enters and organizes within $\mathrm{SN}$ before reaching the central executive network including the prefrontal cortices is still a mystery. Previous electrophysiological studies focused on individual nodes of $\mathrm{SN}$, either on dACC or rAI, have reports of conflicting findings of the earliest cortical activity within the network. Functional magnetic resonance imaging (fMRI) studies are not able to answer these questions in the time-scales of human sensory perception and decision-making. Here, using clear and noisy face-house image categorization tasks and human scalp electroencephalography (EEG) recordings combined with source reconstruction techniques, we study when and how oscillatory activity organizes SN during a perceptual decision. We uncovered that the beta-band (13 - $30 \mathrm{~Hz})$ oscillations bound $\mathrm{SN}$, became most active around $100 \mathrm{~ms}$ after the stimulus onset and the $\mathrm{rAI}$ acted as a main outflow hub within SN for easier decision making task. The SN activities (Granger causality measures) were negatively correlated with the decision response time (decision difficulty). These findings suggest that the $\mathrm{SN}$ activity precedes the executive control in mediating sensory and cognitive processing to arrive at visual perceptual decisions. 


\section{Introduction}

The salience network (SN), consisting of the right anterior insula (rAI), left anterior insula (1AI) and dorsal anterior cingulate cortex (dACC) (Ham et al., 2013; Seeley et al., 2007), responds to behaviorally salient events (Seeley et al., 2007). It plays a crucial role in integrating sensory stimuli to initiate cognitive control (Menon and Uddin, 2010), to implement and maintain task sets (Dosenbach et al., 2006), and to coordinate behavioral responses (Medford and Critchley, 2010). When and how a sensory signal enters and organizes within $\mathrm{SN}$ in a sensory-driven, goal-directed task is not understood. Such understanding can help predict impending perceptual decisions and task executions that involve the prefrontal cortex.

There are two main competing theories that explain the possible 'driving hub' of the SN. First theory proposes that the dACC monitors performance and signals the need for behavioral adaptation (Ridderinkhof et al., 2004). Activity in the dACC signals the need for enhanced cognitive control, and interactions between the $\mathrm{dACC}$ and the lateral prefrontal structures implement subsequent behavioral changes (Egner, 2009; Ridderinkhof et al., 2004). In contrast, the second theory suggests that the rAI is a 'cortical outflow hub' of the $\mathrm{SN}$ and it coordinates a change in activity across multiple neurocognitive networks, such as the default mode network (DMN) and central executive network (CEN) (Chand and Dhamala, 2015; Bonnelle et al., 2012; Menon and Uddin, 2010; Sridharan et al., 2008). Diffusion tensor imaging (DTI) study has demonstrated that the structural integrity of the white matter connection between the rAI and the dACC predicts behavioral and physiological abnormalities after traumatic brain injury (Bonnelle et al., 2012). Previous investigations using blood oxygenation level-dependent (BOLD) 
changes in functional magnetic resonance imaging (fMRI) showed that the rAI, not the dACC, drives the SN (Ham et al., 2013; Sridharan et al., 2008) and further suggested that a change in the effective connectivity of the dACC was associated with behavioral adaptation (Ham et al., 2013). As BOLD hemodynamic responses are sluggish, it might in fact include processes that happen on longer time-scale (seconds) and, if so, the 'driving hub' of the SN might even change in millisecond time-scale of neuronal activities. The studies mentioned above (Debener et al., 2005; Egner, 2009; Ham et al., 2013; Sridharan et al., 2008) had reports of conflicting findings of the earliest cortical activity. Therefore, how a sensory signal enters $\mathrm{SN}$ and organizes within before reaching the prefrontal cortex for central executive processing in the time-scales of human sensory perception and cognition has remained as a mystery. We seek to resolve these conflicting reports considering both anterior insulae and dACC in millisecond time-scale. In particular, how the cortical areas of the SN interact, what the temporal flow of underlying overall activity in these cortical areas is, and what frequency band(s) of information flow binds the SN are largely unknown.

As the dACC, rAI and 1AI are often co-activated, it had been hard to disentangle their causal features (Ham et al., 2013), specifically on longer time-scale measures such as from fMRI. In this study, we recorded human scalp electroencephalography (EEG), reconstructed source waveforms and investigated the causal relationships between the areas of the SN using spectral Granger causality (GC) (Dhamala et al., 2008a; Dhamala et al., 2008b). In this EEG experiment, we used the standard face-house image categorization tasks and studied the temporal evolution of activity in the salience nodes and the patterns of oscillatory network activity flow binding SN nodes in a network. 
Adding noise to clear images, three noise levels of stimuli were created to examine whether a difficult task (or difficult decision) modulates the network activity flow.

\section{Materials and methods}

Participants. Twenty-six neurologically healthy human volunteers (21 males, 5 females) of age ranged from twenty-two to thirty-eight years (mean: 26.3 years, standard deviation: 4.7 years) participated for this study. A written informed consent was collected from the participants prior to data collection. The experimental protocol was approved from Institutional Review Board of Georgia State University. Three participants were excluded from the final analyses because of behavior performance and/or unmanageable artifacts and noise present in their EEG data.

Stimuli. We used total twenty-eight images of faces and houses (14 images of each category). Face images were from the Ekman series (Ekman and Friesen, 1976). Fast Fourier transforms (FFT) of these images were computed, providing twenty-eight magnitude and twenty-eight phase matrices. The average magnitude matrix of this set was stored. Stimulus-images were produced from the inverse FFT (IFFT) of average magnitude matrix and individual phase matrices. The phase matrix used for the IFFT was a linear combination of the original phase matrix computed during the forward Fourier transforms and a random Gaussian noise matrix. The resulting images were equalized for luminance and contrast as in the prior studies (Heekeren et al., 2004; Heekeren et al., 2008; Rainer and Miller, 2000). Finally, the stimuli consisted of three different noiselevels: $0 \%, 40 \%$ and $55 \%$ (i.e., clear stimuli, $40 \%$ noisy stimuli, and $55 \%$ noisy stimuli). Those steps were performed using Matlab scripts. The E-Prime 2.0 software was used to display the stimuli and control the task sequences. 
Experimental design. Prior to experimental task, the participants were briefly explained about the task paradigm. Participant sat in a dark room with the only source of light from the experimenter's computer screen. The same computer screen with the same display settings was used throughout the experiment. However, we did not explicitly calculate voltage/luminance functions for gamma correction as specified by the relations between RGB (red-blue-green) and luminance values, which are device dependent. The stimulus viewing distance was $\sim 60 \mathrm{~cm}$ (chin rest). Fig. 1 shows a schematic of experimental paradigm used. Experiment consisted of 4 blocks with 168 trials in each block. The stimuli were randomized but balanced across blocks in presentation. The experiment consisted of total 672 trials with 224 trials for each noise level. On each trial, a small fixation cross ('+' in the middle of the screen) was presented for $500 \mathrm{~ms}$. Then a stimulus was presented for $150 \mathrm{~ms}$, followed by black screen with question mark ('?’) for $1500 \mathrm{~ms}$ during which time participants were allowed to indicate their decision (either face or house) by keyboard button press. The responses after that delay were considered incorrect.

***Fig. 1 about here***

Data acquisition and preprocessing. EEG data were acquired with a 64-channel EEG system from Brain Vision LLC (http://www.brainvision.com). Analog signal was digitized at $500 \mathrm{~Hz}$. The impedances of each electrode were kept below $10 \mathrm{k} \Omega$, and the participants were asked to minimize blinking, head movements, and swallowing. EEG data were band-pass filtered between 1 and $100 \mathrm{~Hz}$, and notch filtered to remove $60 \mathrm{~Hz}$ AC-line noises. The eyes blinkings were removed using independent component analysis (ICA)-based ocular correction. Data from bad electrodes were discarded and replaced, 
when appropriate, by spatial interpolation from the neighboring working electrodes. These preprocessing steps were done using Brain Vision Analyzer 2.0 (http://www.brainproducts.com).

Data analysis. The preprocessed EEG data were analyzed in the following main steps:

(1) Computation of ERPs: Continuous EEG data were segmented into trials of $300 \mathrm{~ms}$ duration (post-stimulus: 0 to $300 \mathrm{~ms}$ ) based on the stimulus onset times as a reference. The trials that had three standard deviations below or above the global mean across time in each subject were considered as outliers (Junghofer et al., 2000) and they were discarded from the subsequent analysis.

(2) LORETA EEG-sources and single-trials source waveforms reconstruction: All correct trials (ERPs for correct percept) from all three conditions were grand averaged and imported to BESA software version 5.3.7 (www.besa.de) to reconstruct EEG sources. We used the low resolution electromagnetic tomography (LORETA) (Pascual-Marqui et al., 1999; Pascual-Marqui et al., 1994), which is also referred as Laplacian weighted minimum norm, to reconstruct the EEG sources. LORETA is an extensively used source localization technique in EEG studies for both cortical and deep brain structures (Clemens et al., 2010; Herrmann et al., 2005; Jones and Bhattacharya, 2012; Thatcher et al., 2014; Velikova et al., 2010), including insula and hippocampus (Jones and Bhattacharya, 2012; Thatcher et al., 2014; Velikova et al., 2010). Depth weighting strategy implemented in LORETA overcomes the problem of surface-restricted localization methods, such as minimum norm estimates (MNE) (Michel et al., 2004; Painold et al., 2011; Pascual-Marqui et al., 1999). LORETA computes inverse solution at 2394 voxels with spatial resolutions of $7 \mathrm{~mm}$ in the Talairach Atlas (Pascual-Marqui et 
al., 1999; Pascual-Marqui et al., 1994). It is based on the assumption that the smoothest of all possible neural activity distributions is the most plausible one. This assumption is also supported by electrophysiology, where neighboring neuronal populations show highly correlated activity while EEG-LORETA results are the activity rendered by neighboring voxels with maximally similar activity (Haalman and Vaadia, 1997; Herrmann et al., 2005; Michel et al., 2004). Since functionally very distinct areas can be anatomically very close (e.g., the medial parts of the two hemispheres), LORETA can produce the results that include the two hemispheres (Fig. S1: activity in V1). Therefore, the results should be interpreted with caution and simultaneous EEG-fMRI recordings could be a good choice in such case.

Locations of sources can be constrained to the cortical surface and their orientations perpendicular to the local cortical surface based on neurophysiological information that the sources of EEG are postsynaptic currents in cortical pyramidal cell, and that the direction of these currents is perpendicular to the cortical surface (Dale and Sereno, 1993; Hamalainen et al., 1993). Peak activities of these sources - the SN nodes - were marked as the network nodes for connectivity analyses. Using single-trials EEG data, we fitted dipoles at locations of peak activation of localized sources of SN - the rAI, 1AI, and dACC based on our hypothesis - with dipole orientation presented in table 1 . The single-trials source signals were then extracted using a four-shell spherical head model and a regularization constant of $1 \%$ for the inverse operator as done previously by our group (Adhikari et al., 2014). These source signals were used for the connectivity analyses. 
(3) Power and Granger causality spectral analyses. The power spectra can be computed using parametric and nonparametric approaches (Chand and Dhamala, 2014; Dhamala et al., 2008a; Dhamala et al., 2008b). Granger causality (GC) spectral analyses are used to examine the strengths, directions, and frequencies of interactions between dynamic processes. GC spectral method is a part of spectral interdependency methods (Dhamala, 2014). The measures of spectral interdependency are derived from the time series recordings of dynamic systems either by using autoregressive modeling (parametric method), or by using direct Fourier or wavelet transforms (nonparametric method) (Dhamala et al., 2008a; Dhamala et al., 2008b). For a pair of multivariate stationary processes (1 and 2), there are three measures that characterize the spectral interdependency between these processes: total interdependence $\left(\mathrm{M}_{1,2}\right)$, $\mathrm{GC}$ (one-way effect or directional influence from the first process to the second process, $\mathrm{M}_{1 \rightarrow 2}$, or from the second to the first, $\mathrm{M}_{2 \rightarrow 1}$ ) and instantaneous causality (measure of reciprocity, $\mathrm{M}_{1.2}$ ). In general, the total interdependence is the sum of directional influences and instantaneous causality frequency by frequency $\left(\mathrm{M}_{1,2}=\mathrm{M}_{1 \rightarrow 2}+\mathrm{M}_{2 \rightarrow 1}+\mathrm{M}_{1.2}\right)$. The spectral interdependency measures are derived from the spectral matrix (S), and/or from the transfer function $(\mathrm{H})$ and noise covariance matrix $(\Sigma)$, which can be estimated by the parametric (prediction model building) (Ding et al., 2006) or nonparametric (model-free) approaches applied to these time series (Dhamala et al., 2008a; Dhamala et al., 2008b). $\sum$ is computed from the residual errors of the prediction models and the transfer function matrix $\mathrm{H}$ is constructed from the matrix inverse of the Fourier transforms of the coefficients in the prediction models. S, $\mathrm{H}$ and $\sum$ can also be estimated by using the nonparametric spectral methods (Dhamala et al., 2008a; Dhamala et al., 2008b) without 
explicitly fitting the time series $\mathrm{X}_{1}(\mathrm{t})$ and $\mathrm{X}_{2}(\mathrm{t})$ in autoregressive models. The GC spectrum from the second time series $X_{2}$ to the first time series $X_{1}$ (i.e., 2 to 1 ) at a frequency (f) is defined as

$$
M_{2 \rightarrow 1}(f)=-\ln \left(1-\frac{\left(\Sigma_{22}-\Sigma_{12}^{2} / \Sigma_{11}\right)\left|H_{12}(f)\right|^{2}}{S_{11}(f)}\right)
$$

Because of the unknown theoretical distributions of spectral GC, establishing statistical significance in these measures from experimental time series requires data resampling (surrogate) methods such as random permutation method (Adhikari et al., 2013; Brovelli et al., 2004; Seth, 2010).

In EEG-source waveforms, the frequency-specific causal outflow $(F)$ at a node $i$ can be defined as:

$F_{i}=\frac{1}{N-1} \sum_{j}^{N}\left(M_{i \rightarrow j}-M_{j \rightarrow i}\right)$

Here, for the three nodes, $\mathrm{j}$ can be 1,2 , and 3 . If we assign, for example, the $\mathrm{rAI}=1$ (the first node), $\mathrm{dACC}=2$ (the second node), and $\mathrm{AII}=3$ (the third node), then GC causal

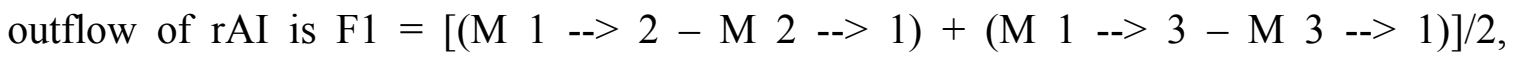
where M1 --> 2 is GC from the first node to second node, and M2 --> 1 is GC from the second node to first node. Similarly, GC outflows can be defined for the dACC and 1AI. The model order appropriate for the data turned out to be 4 , which was determined by comparing the power computed from parametric and nonparametric approaches at different model orders, and picking the model order that rendered the lowest power difference between two approaches. Since we were interested in investigating the salience node and network activities across time, we segmented the trials in four time frames. The 
source activity of the AIs and dACC peaked around 75-145 ms (as presented in Table 1 and Fig S1). We use this time frame in a segment and consider other equal intervals before and after it. Thus, we had four time segments. The model orders were also further computed at all four segments and the appropriate model order was found out to be common across all segments. We computed the power and GC spectra from source waveforms of SN nodes in four consecutive time frames (TFs) named as TF1: $0 \mathrm{~ms}$ to 75 ms, TF2: $75 \mathrm{~ms}$ to $150 \mathrm{~ms}$, TF3: $150 \mathrm{~ms}$ to $225 \mathrm{~ms}$, and TF4: $225 \mathrm{~ms}$ to $300 \mathrm{~ms}$. The threshold value of GC, for statistical significance, was computed from surrogate data by using permutation tests and a gamma-function fit (Adhikari et al., 2014; Blair and Karniski, 1993) under a null hypothesis of no interdependence at the significance level p $<10^{-4}$. The causal outflow at a node (See Eq. 2) is computed as the total GC flowing out from a node minus total GC flowing in to that node in beta band (13-30 Hz).

Brain-behavioral correlation. The response time (RT) of each participant for each stimulus was recorded. To see the brain-behavioral correlation with the increase in noise level in stimuli, RTs were converted into z-scores and plotted with GC. Relationship between GC and RT was tested using both Spearman's rank correlation and Pearson's correlation. If $\mathrm{p}<0.05$ for both, the correlation was considered significant. The results reported here are in terms of Spearman's rank correlation.

\section{Results}

Behavioral results. The mean performance percent, defined as a ratio of the number of correct responses to the total number of responses multiplied by hundred and averaged over all participants, was the highest for stimuli with $0 \%$ noise level (with mean: $96.80 \%$ and standard deviation: $0.71 \%$ ) compared to the stimuli with $40 \%$ noise level (mean: 
92.68\%, standard deviation: $1.54 \%$ ) and 55\% noise level (mean: $69.67 \%$, standard deviation: 2.74\%). Repeated measures ANOVA (Sheskin, 2003) showed a significant effect of noise levels (task difficulty) on the performance $(\mathrm{F}(2,44)=150.43, \mathrm{p}=$ $0.000003)$ and the response time $(\mathrm{RT})(\mathrm{F}(2,44)=132.74, \mathrm{p}=0.000000)$. Pair t-test post hoc analyses followed by false discovery rate (FDR) multiple comparisons (Benjamini and Hochberg, 1995) further revealed that performance significantly decreased with the increase in noise level ( $\mathrm{p}<10-3$; FDR-corrected). The mean RT-the time taken to indicate the decision by pressing keyboard button press and averaged over all participants — was lower for the stimuli with $0 \%$ noise level (mean: $434.02 \mathrm{~ms}$, standard deviation: $22.09 \mathrm{~ms}$ ) compared to the stimuli with $40 \%$ noise level (mean: $484.28 \mathrm{~ms}$, standard deviation: $22.66 \mathrm{~ms}$ ) and 55\% noise level (mean: $565.70 \mathrm{~ms}$, standard deviation: $25.73 \mathrm{~ms})$. Pair t-test post hoc analyses followed by FDR multiple comparisons further illustrated that the RT significantly increased with the increase in noise level ( $\mathrm{p}<10-6$; FDR-corrected) as shown in Fig. 2.

*** Fig. 2 about here**

\section{Electrophysiological (Brain) results}

Activities of SN nodes. The average ERPs for correct decisions were used to compute localized sources (inverse solutions) in LORETA (Pascual-Marqui et al., 1999; Pascual-Marqui et al., 1994). Fig. 3 shows the locations of the peak source activity (marked by cross-hairs) in time (first row), and the locations and orientations of fitted dipoles in the $\mathrm{SN}$ nodes (second row) to obtain the single-trials source waveforms.

*** Fig. 3 about here*** 
Activation in the $\mathrm{SN}$ nodes started at $\sim 76 \mathrm{~ms}$ after the stimulus onset. Maximum peak activations occurred at $\sim 84 \mathrm{~ms}$ in the rAI and 1AI (BA47/13), which was followed by activation in the dACC (BA32) at $\sim 98$ ms. Besides activations in the SN nodes, we also observed activation in the visual area (BA17/18: $\left.\mathrm{V}_{1} / \mathrm{V}_{2}\right)$, the ventral temporal cortex (BA37: the right fusiform face area (FFA) and the left parahippocampal place area (PPA)), and in the left dorsolateral prefrontal cortex (DLPFC) (BA9) (Fig. S1). We limited our study to the SN nodes and therefore performed the dipole fittings at those SN nodes. Table 1 lists the source locations, dipole orientations in the source model, and dominant activation timeframes of the SN nodes (Table $\mathrm{S} 1$ for activation timeframes of other brain areas). The dipoles fitted at the locations and orientations explained approximately $80 \%$ of the variance in the EEG signal for trials with correct responses.

\section{***Table 1 about here***}

SN for clear stimuli. Power spectra computed in four consecutive timeframes TF1: $0 \mathrm{~ms}$ to $75 \mathrm{~ms}$, TF2: $75 \mathrm{~ms}$ to $150 \mathrm{~ms}$, TF3: $150 \mathrm{~ms}$ to $225 \mathrm{~ms}$, and TF4: $225 \mathrm{~ms}$ to $300 \mathrm{~ms}$ - at the rAI, 1AI, and dACC showed peak activity in beta $(24 \mathrm{~Hz}$ peak $)$ band when the participants viewed clear stimuli (see Figs. S2, S3). Fig. 4A shows power spectra comparison within nodes of the SN among TF1, TF2, TF3 and TF4. Repeated measures ANOVA calculations demonstrated a significant effect on overall power spectra across time frames $(\mathrm{F}(3,44)=128.43, \mathrm{p}=0.000000)$. Pair $\mathrm{t}$-test post hoc analyses followed by FDR multiple comparisons further revealed that overall power spectra significantly changed over the time frame and had significantly higher magnitude 
in TF2 compared to other timeframes as shown in Fig. 4B $(*$ indicates $\mathrm{p}<0.001$; FDRcorrected). GC spectra were computed to assess the oscillatory network interactions between the SN nodes.

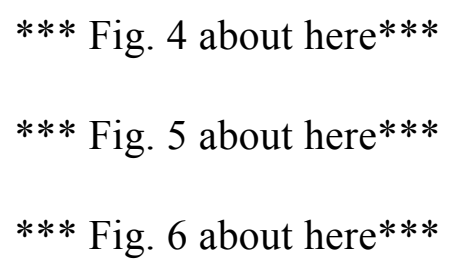

Fig. 5 presents GC spectra as a function of frequency, where horizontal lines represent statistically significant threshold value. Beta band network interactions between the SN nodes enhanced in TF2 (Fig. 5: second column) compared to the rest of TFs (other columns). Repeated measures ANOVA calculations showed a significant effect on overall GC spectra across time frames $(F(3,44)=51.68, p=0.0002)$. Pair t-test post hoc analyses followed by FDR multiple comparisons further evaluated that overall GC spectra significantly changed over the time frame and had a higher magnitude in TF2 compared to other timeframes as shown in Fig. 6A (* indicates $\mathrm{p}<0.01$; FDRcorrected). We calculated GC net outflow at each node of the SN in TF2, applied the repeated measures ANOVA, and found that the net outflows were significantly different among the nodes $(\mathrm{F}(2,44)=122.12, \mathrm{p}=0.000000)$. Pair t-test post hoc analyses followed by FDR further revealed that the net outflows were significantly different over the SN nodes as displayed in Fig. 6B (* indicates $\mathrm{p}<0.00001$; FDR-corrected). Importantly, those comparisons of the net outflows suggested that the rAI as a main 'cortical outflow hub' and the dACC as a main 'cortical inflow hub' within the SN (Fig. $6 B)$. 
Effect of task-difficulty in the SN activity. Power spectra were computed for different timeframes TF1, TF2, TF3, and TF4 at the rAI, 1AI, and dACC when participants viewed the stimuli with $40 \%$ and 55\% noise-levels (Figs. S4, S6). Power spectra calculations also showed a peak activity in beta band $(\sim 24 \mathrm{~Hz}$ peak). GC spectra were computed to assess the oscillatory network interactions between the SN nodes.

*** Fig. 7 about here ${ }^{* * *}$

Beta band network interactions between the SN nodes were suppressed for noisy stimuli compared to clear stimuli in the TF2 (see second columns of Figs. 5, S5, S7). Overall causal interactions among the SN nodes were compared between noiselevels by repeated measures ANOVA to assess significance level of task difficulty. Repeated measures ANOVA showed a significant effect of noise (task difficulty) on overall causal interactions among the SN nodes $(F(2,44)=215.02, p=0.00000008)$. Pair t-test post hoc analyses followed by FDR multiple comparisons further revealed that the overall causal interactions significantly decreased with increase in noise level $(\mathrm{p}<10-3$; FDR-corrected) (Fig. 7).

Brain-behavior correlation. The difficulty levels-expressed in terms of behavior response times - were found to be significant and negatively correlated with the measures of network activity for all possible connections between cortical areas of the $\mathrm{SN}$, except for the dACC to 1AI flow, in the TF2 $(75 \mathrm{~ms}-150 \mathrm{~ms})$. The correlation coefficient (r) and the corresponding p-value of all possible connections are presented in Fig. 8 (see Figs. S8, S9, and S10 for other TFs).

*** Fig. 8 about here*** 


\section{Discussion}

Behaviorally important events are responsible to activate the SN (Menon and Uddin, 2010; Seeley et al., 2007). The SN nodes - the rAI, 1AI and dACC - are often coactivated in fMRI BOLD responses (Ham et al., 2013; Ullsperger et al., 2010) and therefore make harder to clearly identify their distinct functional roles within a network. In this study, using EEG recordings and perceptual decision-related reconstructed EEG sources, we looked at the temporal changes of network activity flow within SN. Using spectral GC analyses, we found that beta $(\sim 24 \mathrm{~Hz})$ oscillation bound these nodes in the SN. The beta power and beta causal interactions were significantly higher at the nodes and network in the time frame $75 \mathrm{~ms}$ to $150 \mathrm{~ms}$ compared to other time frames. The analysis of the net beta causal outflow (out - in causality) patterns showed that the rAI played as a main 'cortical outflow hub' of the SN consistent with previous fMRI studies of the SN (Ham et al., 2013; Sridharan et al., 2008). We also found that the beta causal interactions within the SN were significantly suppressed with the task difficulty (noise level), but the spectral power was opposite (Fig. 7 and Fig. S11). The causal outflow was negatively correlated with the response time (Fig. 8).

Activities of the SN nodes. We found that the key nodes of SN-the rAI, 1AI and dACC - activated at around $100 \mathrm{~ms}$ after stimulus onset. These brain regions were demonstrated also being activated for a variety of tasks in previous fMRI investigations (Ham et al., 2013; Menon and Uddin, 2010; Seeley et al., 2007). Electrophysiological recordings combined with source localization techniques (Debener et al., 2005; Dehaene et al., 1994) reported that the dACC responds to the salient events, such as error detection, in the time frame of $80 \mathrm{~ms}-110 \mathrm{~ms}$. The timing of dominant activation of the 
dACC in our study was consistent with those studies. Similarly, the AI was reported being activated at $\sim 60 \mathrm{~ms}$ after stimulus onset, however it was in a thermo-sensory domain (Craig et al., 2000). Previous study on monkeys had found that the AI activated at $\sim 65$ ms after stimulus onset, however it was in an auditory domain (Remedios et al., 2009). Our findings and previous reports taken together therefore suggested the overall time frame of activations of $\mathrm{SN}$ nodes.

Oscillatory power and network activity. Power spectra estimated at the rAI, 1AI and dACC showed prominent peak activity in beta ( $\sim 24 \mathrm{~Hz}$ peak) band when the participants viewed clear stimuli. Beta oscillations were also at work for noisy stimuli. This band of oscillatory activity was consistent with ones observed in previous EEG studies of multisensory (Hipp et al., 2011) and somatosensory (Adhikari et al., 2014) perceptions for different brain regions. A recent study (Castelhano et al., 2014) also showed that the AI activity especially in lower gamma (or higher beta) is associated with perceptual decisions.

GC spectral analyses demonstrated that inter-areal brain synchronization and interactions within the $\mathrm{SN}$ are mediated by enhanced $\sim 24 \mathrm{~Hz}$ (beta band) neural oscillations. Information flow at $\sim 24 \mathrm{~Hz}$ was dominantly feedforward from sensory areas - the rAI and the $1 \mathrm{AI}$ - to the dACC, similar to the propagation of average cortical activity, probably reflecting sensory-driven processes. This supports the model of feedforward hierarchical integration process from sensation to those ultimately ends up into action (Mazurek et al., 2003; Smith and Ratcliff, 2004). Previous investigations that consider nodes of the SN have divided into two competing groups. One group supports that activity in the dACC implies an enhanced cognitive control (Ridderinkhof et al., 
2004), and further interactions of the dACC with the lateral prefrontal structures implement subsequent behavioral changes (Egner, 2009; Ridderinkhof et al., 2004). Electrophysiological studies (Debener et al., 2005; Dehaene et al., 1994) suggested that the dACC provides the first cortical signal used for salient events, such as error detection. Other studies support that the rAI drives the SN, partly because the rAI is shown to be structurally and functionally connected to a wide range of cortical regions involved in various aspects of cognitive control. The rAI is functionally connected to networks responsible for adaptive behavior, including the SN (Seeley et al., 2007), as well as other parts of the fronto-parietal control network (Vincent et al., 2008). DTI tractography has demonstrated that this cortical area has direct white matter connections to other key regions within these networks, including the dACC (van den Heuvel et al., 2009), the inferior parietal lobe (Uddin et al., 2010), and the temporo-parietal junction (Kucyi et al., 2012) making the insula well placed to perform its putative role of evaluating (Uddin et al., 2010), reorienting attention (Ullsperger et al., 2010), and switching between cognitive resources in response to salient events (Uddin and Menon, 2009). While there is an ongoing division based on previous studies, our results demonstrated that the rAI is a main 'cortical outflow hub' and the dACC is a main 'cortical inflow hub' of the SN (Fig. $6 \mathrm{~B})$. We provided this evidence showing the milliseconds time-scale evolution of beta activity in perceptual decision-making task. As cortical circuits implementing cognitive processes might engage in highly recurrent interactions (Wang, 2008) mediated by bidirectional cortico-cortical connections (Felleman and Van Essen, 1991), our results also demonstrated that beta networks are bidirectional such as in rAI-lAI pair, however a dominant flow is from the rAI to 1AI in the time frame of highest activity. Our results 
demonstrated that the overall spectral power increased with the increase in noise levels (Fig. S11), however the GC connectivity strengths showed the opposite (Figs. 7, 8). Previous studies in disease (Zheng-yan, 2005) and health (Hipp et al., 2011) also report the similar features of beta-band activity that local activity (power) is positively correlated and large-scale network activity (coherence) is negatively correlated with difficulty levels of task. A recent report (Castelhano et al., 2015) also demonstrates a dissociated role between amplitude (power) measures and coherence synchrony. Those reports and our findings taken together therefore suggest that detailed neural mechanisms for such dissociation might be important for future research. The suppression in GC connectivity might be due to less information flow from the sensory regions to the dACC when stimuli were degraded. Since beta oscillations have recently been observed for a better performance (or, accuracy) during decision-making processes (Hipp et al., 2011; Siegel et al., 2012; Siegel et al., 2011), this suppressed information flow might implicate the underlying neuronal mechanisms of the $\mathrm{SN}$ that has also been indeed behaviorally reflected for an elevated difficulty level (Fig. 2A). The anatomical inter-areal and laminar neural circuitry in the brain might support the occurrence of such oscillatory activities and their modulations in cognitive tasks (Buzsaki and Wang, 2012).

To summarize, the present study enhances our understanding of the SN activity, with regards to the temporal evolution of averaged evoked potentials in the nodes and an oscillatory network that appear to organize evoked activity across the SN. We found that SN became most active at around $100 \mathrm{~ms}$ after the stimulus onset; the beta-band $(13-30$ $\mathrm{Hz}$ ) oscillations bound them in a network and the rAI acted as a main outflow hub within $\mathrm{SN}$. The SN activities (GC measures) were negatively correlated with the decision 
response time (decision difficulty). These findings provide important insights on how sensory information enters and organizes in the $\mathrm{SN}$ before reaching the prefrontal cortex for decision-making.

\section{Acknowledgements}

We would like to thank Eli Goshorn for writing Matlab scripts to systematically add noise to visual images, Dr. Bidhan Lamichhane for the help in data collection, and Dr. Bhim Adhikari for various discussions on dipole-modeling for EEG implemented in a recent study (Adhikari et al., 2014). A National Science Foundation CAREER award (BCS 0955037) to the author MD financially supported this work.

\section{References}

Adhikari, B.M., Epstein, C.M., Dhamala, M., 2013. Localizing epileptic seizure onsets with Granger causality. Phys Rev E 88, 030701(R).

Adhikari, B.M., Sathian, K., Epstein, C.M., Lamichhane, B., Dhamala, M., 2014. Oscillatory activity in neocortical networks during tactile discrimination near the limit of spatial acuity. Neuroimage 91,300-310.

Benjamini, Y., Hochberg, Y., 1995. Controlling the false discovery rate: A practical and powerful approach to multiple testing. J Royal Statistical Society B (Methodological), 289-300.

Blair, R.C., Karniski, W., 1993. An alternative method for significance testing of waveform difference potentials. Psychophysiol 30, 518-524. 
Bonnelle, V., Ham, T.E., Leech, R., Kinnunen, K.M., Mehta, M.A., Greenwood, R.J., Sharp, D.J., 2012. Salience network integrity predicts default mode network function after traumatic brain injury. Proc Natl Acad Sci U S A 109, 4690-4695.

Brovelli, A., Ding, M., Ledberg, A., Chen, Y., Nakamura, R., Bressler, S.L., 2004. Beta oscillations in a large-scale sensorimotor cortical network: directional influences revealed by Granger causality. Proc. Natl. Acad. Sci. U. S. A. 101, 9849-9854.

Buzsaki, G., Wang, X.J., 2012. Mechanisms of gamma oscillations. Annu Rev Neurosci 35, 203-225.

Castelhano, J., Duarte, I. C., Wibral, M., Rodriguez, E., Castelo-Branco, M., 2014. The dual facet of gamma oscillations: separate visual and decision making circuits as revealed by simultaneous EEG/fMRI. Human Brain Mapping 35, 5219-5235.

Castelhano, J., Bernardino, I., Rebola, J., Rodriguez, E., Castelo-Branco, M., 2015. Oscillations or synchrony? Disruption of neural synchrony despite enhanced gamma oscillations in a model of disrupted perceptual coherence. J Cogn Neurosci 27(12), 2416-26.

Chand, G.B., Dhamala, M., 2015. Interactions among the brain default-mode, salience and central-executive networks during perceptual decision-making of moving dots. Brain Connectivity, DOI: 10.1089/brain.2015.0379

Chand, G.B., Dhamala, M., 2014. Spectral factorization-based current source density analysis of ongoing neural oscillations. J Neurosci Methods 224, 58-65.

Clemens, B., Bessenyei, M., Fekete, I., Puskas, S., Kondakor, I., Toth, M., Hollody, K., 2010. Theta EEG source localization using LORETA in partial epilepsy patients with and without medication. Clin Neurophysiol 121, 848-858. 
Craig, A.D., Chen, K., Bandy, D., Reiman, E.M., 2000. Thermosensory activation of insular cortex. Nat Neurosci 33, 184 - 190.

Dale, A.M., Sereno, M.I., 1993. Improved localization of cortical activity by combining EEG and MEG with MRI cortical surface reconstruction: A linear approach. J Cogn Neurosci 5, 162-176.

Debener, S., Ullsperger, M., Siegel, M., Fiehler, K., Von Cramon, D.Y., Engel, A.K., 2005. Trial-by-trial coupling of concurrent electroencephalogram and functional magnetic resonance imaging identifies the dynamics of performance monitoring. J Neurosci $25,11730-11737$.

Dehaene, S., Posner, M.I., Tucker, D.M., 1994. Localization of a neural system for errordetection and compensation. Psychol Sci 5, 303-305.

Dhamala, M., 2014. Spectral Interdepdndency Methods. Enclyclopedia of Computational Neuroscience, 1-6.

Dhamala, M., Rangarajan, G., Ding, M., 2008a. Analyzing information flow in brain networks with nonparametric Granger causality. Neuroimage 41, 354-362.

Dhamala, M., Rangarajan, G., Ding, M., 2008b. Estimating Granger Causality from Fourier and Wavelet Transforms of Time Series Data. Physical Review Letters 100.

Ding, M., Chen, Y., Bressler, S., 2006. Handbook of Time Series Analysis: Recent Theoretical Developments and Applications. (Eds.) B. Schelter, M. Winterhadler, J. Timmers, Wiley-VCH, Berlin, 2006:2437-2459.

Dosenbach, N.U., Visscher, K.M., Palmer, E.D., Miezin, F.M., Wenger, K.K., Kang, H.C., Burgund, E.D., Grimes, A.L., Schlaggar, B.L., Petersen, S.E., 2006. A core system for the implementation of task sets. Neuron 50, 799-812. 
Egner, T., 2009. Prefrontal cortex and cognitive control: motivating functional hierarchies. Nat Neurosci 12, 821-822.

Ekman, P., Friesen, W.V., 1976. Pictures of facial affect. Palo Alto (CA): Consulting Psychologists Press.

Felleman, D.J., Van Essen, D.C., 1991. Distributed hierarchial processing in the primate cerebral cortex. Cereb Cortex 1, 1-47.

Haalman, I., Vaadia, E., 1997. Dynamics of neuronal interactions: relation to behavior, firing rates, and distance between neurons. Human Brain Mapping 5, 249-253.

Ham, T., Leff, A., de Boissezon, X., Joffe, A., Sharp, D.J., 2013. Cognitive control and the salience network: an investigation of error processing and effective connectivity. J Neurosci 33, 7091-7098.

Hamalainen, M., Hari, R., Ilmoniemi, R.J., Knuutila, J., Lounasmaa, O.V., 1993. Magnetoencephalography- theory, instrumentation, and applications to noninvasive studies of the working human brain. Rev. Mod. Phys. 65, 413-497.

Heekeren, H.R., Marrett, S., Bandettini, P.A., Ungerleider, L.G., 2004. A general mechanism for perceptual decision-making in the human brain. Nature 431, 859-862.

Heekeren, H.R., Marrett, S., Ungerleider, L.G., 2008. The neural systems that mediate human perceptual decision making. Nat Rev Neurosci 9, 467-479.

Herrmann, M.J., Ehlis, A.C., Muehlberfer, A., Fallgatter, A.J., 2005. Source localization of early stages of face processing. Brain Topography 18, 77-85.

Hipp, J.F., Engel, A.K., Siegel, M., 2011. Oscillatory synchronization in large-scale cortical networks predicts perception. Neuron 69, 387-396. 
Jones, R., Bhattacharya, J., 2012. Alpha activity in the insula accompanies the urge to neutralize in sub-clinical obsessive-compulsive participants. J Behav Addict 1, 96105.

Junghofer, M., Elbert, T., Tucker, D.M., Rockstroh, B., 2000. Statistical control of artifacts in dense array EEG/MEG studies. Psychophysiol 37, 523-532.

Kucyi, A., Moayedi, M., Weissman-Fogel, I., Hodaie, M., Davis, K.D., 2012. Hemispheric asymmetry in white matter connectivity of the temporoparietal junction with the insula and prefrontal cortex. PLoS One 7, e35589.

Mazurek, M.E., Roitman, J.D., Diterich, J., Shadlen, M.N., 2003. A role for neural integrators in perceptual decision making. Cereb Cortex 13, 1257-1269.

Medford, N., Critchley, H.D., 2010. Conjoint activity of anterior insular and anterior cingulate cortex: awareness and response. Brain Struct Funct 214, 535-549.

Menon, V., Uddin, L.Q., 2010. Saliency, switching, attention and control: a network model of insula function. Brain Struct Funct 214, 655-667.

Michel, C.M., Murray, M.M., Lantz, G., Gonzalez, S., Spinelli, L., Grave de Peralta, R., 2004. EEG source imaging. Clin Neurophysiol 115, 2195-2222.

Painold, A., Anderer, P., Holl, A.K., Letmaier, M., Saletu-Zyhlarz, G.M., Saletu, B., Bonelli, R.M., 2011. EEG low-resolution brain electromagnetic tomography (LORETA) in Huntington's disease. J Neurol 258, 840-854.

Pascual-Marqui, R.D., Lehmann, D., Koenig, T., Kochi, K., Merlo, M.C.G., Hell, D., Koukkou, M., 1999. Low resolution brain electromagnetic tomography (LORETA) functional imaging in acute, neuroleptic-naive, first-episode, productive schizophrenia. Psychiatry Research Neuroimaging 90, 169-479. 
Pascual-Marqui, R.D., Michel, C.M., Lehmann, D., 1994. Low resolution electromagnetic tomography: A new method for localizing electrical activity in the brain. Int J Psychophysiol, 49-65.

Rainer, G., Miller, E.K., 2000. Effects of visual experience on the representation of objects in the prefrontal cortex. Neuron 27, 179-189.

Remedios, R., Logothetis, N.K., Kayser, C., 2009. An auditory region in the primate insular cortex responding preferentially to vocal communication sounds. J Neurosci 29, 1034-1045.

Ridderinkhof, K.R., Ullsperger, M., Crone, E.A., Nieuwenhuis, S., 2004. The role of the medial frontal cortex in cognitive control. Science 306, 443-447.

Seeley, W.W., Menon, V., Schatzberg, A.F., Keller, J., Glover, G.H., Kenna, H., Reiss, A.L., Greicius, M.D., 2007. Dissociable intrinsic connectivity networks for salience processing and executive control. J Neurosci 27, 2349-2356.

Seth, A.K., 2010. A MATLAB toolbox for Granger causal connectivity analysis J Neurosci Meth 186, 262-273.

Sheskin, D.J., 2003. Handbook of parametric and nonparametric statistical procedures. Chapman \& Hall/CRC Press Third Edition.

Siegel, M., Donner, T.H., Engel, A.K., 2012. Spectral fingerprints of large-scale neuronal interactions. Nat Rev Neurosci 13, 121-134.

Siegel, M., Engel, A.K., Donner, T.H., 2011. Cortical network dynamics of perceptual decision-making in the human brain. Front Hum Neurosci 5, 21.

Smith, P.L., Ratcliff, R., 2004. Psychology and neurobiology of simple decisions. Trends Neurosci 27, 161-168. 
Sridharan, D., Levitin, D.J., Menon, V., 2008. A critical role for the right fronto-insular cortex in switching between central-executive and default-mode networks. Proc Natl Acad Sci U S A 105, 12569-12574.

Thatcher, R.W., North, D.M., Biver, C.J., 2014. LORETA EEG phase reset of the default mode network. Front Hum Neurosci 8, 529.

Uddin, L.Q., Menon, V., 2009. The anterior insula in autism: under-connected and underexamined. Neurosci Biobehav Rev 33, 1198-1203.

Uddin, L.Q., Supekar, K., Amin, H., Rykhlevskaia, E., Nguyen, D.A., Greicius, M.D., Menon, V., 2010. Dissociable connectivity within human angular gyrus and intraparietal sulcus: evidence from functional and structural connectivity. Cereb Cortex 20, 26362646.

Ullsperger, M., Harsay, H.A., Wessel, J.R., Ridderinkhof, K.R., 2010. Conscious perception of errors and its relation to the anterior insula. Brain Struct Funct 214, 629-643.

van den Heuvel, M.P., Mandl, R.C., Kahn, R.S., Hulshoff Pol, H.E., 2009. Functionally linked resting-state networks reflect the underlying structural connectivity architecture of the human brain. Hum Brain Mapp 30, 3127-3141.

Velikova, S., Locatelli, M., Insacco, C., Smeraldi, E., Comi, G., Leocani, L., 2010. Dysfunctional brain circuitry in obsessive-compulsive disorder: source and coherence analysis of EEG rhythms. Neuroimage 49, 977-983.

Vincent, J.L., Kahn, I., Snyder, A.Z., Raichle, M.E., Buckner, R.L., 2008. Evidence for a frontoparietal control system revealed by intrinsic functional connectivity. J Neurophysiol 100, 3328-3342.

Wang, X.J., 2008. Decision making in recurrent neuronal circuits. Neuron 60, 215-234. 
Zheng-yan, J., 2005. Study of EEG power and coherence in patients with mild cognitive impairment during working memory task. JZUS B, 6(12): 1213-1219

\section{Figure captions}

Fig. 1: Experimental design. A) Stimuli with three noise levels, B) task paradigm: stimuli were presented for $150 \mathrm{~ms}$, followed by black screen with question mark ('?') for 1500 ms during which time participants responded with a keyboard button press.

Fig. 2: Behavior responses for all three levels of noise. A) Behavioral accuracy (performance \%) significantly decreased $\left(*\right.$ indicates significant $\mathrm{p}$-value $\left(\mathrm{p}<10^{-3}\right.$; FDR-corrected)), but B) the response time significantly increased with an elevated noise in the stimuli $\left(*\right.$ indicates significant $\mathrm{p}$-value $\left(\mathrm{p}<10^{-6} ;\right.$ FDR-corrected $)$ ).

Fig. 3: Spatiotemporal profiles of peak source-level brain activity. The first row shows 
peak source-level brain activity over the right anterior insula (rAI) and left anterior insula (lAI) at $84 \mathrm{~ms}$, and over the dorsal anterior cingulate cortex (dACC) at $98 \mathrm{~ms}$ (abbreviations: $1=$ left and $\mathrm{r}=$ right; color bar in $\mathrm{nAm} / \mathrm{cm}^{3}$ ), and the second row shows the fitted dipoles on the $\mathrm{SN}$ nodes (abbreviations: $\mathrm{A}=$ anterior, and $\mathrm{P}=$ posterior).

Fig. 4: A) Comparison of power within nodes of the SN among four consecutive time frames (TF1: $0 \mathrm{~ms}$ to $75 \mathrm{~ms}$, TF2: $75 \mathrm{~ms}$ to $150 \mathrm{~ms}$, TF3: $150 \mathrm{~ms}$ to $225 \mathrm{~ms}$, and TF4: $225 \mathrm{~ms}$ to $300 \mathrm{~ms}$ for $0 \%$ noise-level. B) Average power over the SN nodes in TF2 is significantly higher compared to other TFs $(*$ indicates significant $\mathrm{p}$-value (p $<0.001$; FDR-corrected) and n. s. indicates not significant p-value).

Fig. 5: GC spectra of all possible pairs among the rAI, 1AI, and dACC for stimuli with 0\% noise-level at four consecutive time frames (TF1: $0 \mathrm{~ms}$ to $75 \mathrm{~ms}$, TF2: $75 \mathrm{~ms}$ to 150 ms, TF3: $150 \mathrm{~ms}$ to $225 \mathrm{~ms}$, and TF4: $225 \mathrm{~ms}$ to $300 \mathrm{~ms}$ ) mentioned at top of each column.

Fig. 6: A) Comparison of connectivity strengths within nodes of the SN among four consecutive time frames (TF1: $0 \mathrm{~ms}$ to $75 \mathrm{~ms}$, TF2: $75 \mathrm{~ms}$ to $150 \mathrm{~ms}$, TF3: $150 \mathrm{~ms}$ to 225 $\mathrm{ms}$, and TF4: $225 \mathrm{~ms}$ to $300 \mathrm{~ms}$ ) for $0 \%$ noise-level (* indicates significant p-value (p $<0.01$; FDR-corrected) and n. s. indicates not significant p-value). B) Causal outflow calculations at the time frame of highest connectivity strength (TF2) revealed the rAI as a main 'cortical outflow hub' within the SN (* indicates significant $\mathrm{p}$-value $(\mathrm{p}<0.0001 ;$ FDR-corrected $))$.

Fig. 7: Comparison of connectivity strength within nodes of SN among all three noiselevels in the TF2: $75 \mathrm{~ms}$ to $150 \mathrm{~ms}$. The overall connectivity strength is significantly 
suppressed with an elevated noise in the stimuli (* indicates significant p-value $\left(\mathrm{p}<10^{-3} ;\right.$ FDR-corrected) $)$.

Fig. 8: Relation between GC and difficulty level expressed in terms of response time of all three noise-levels in TF2 (75 ms to $150 \mathrm{~ms})$.

\section{Table caption}

Table 1: The anatomical location, dipole orientation and dominant activation timeframe of localized sources of the $\mathrm{SN}$ for correctly perceived stimuli. 
Figures

A

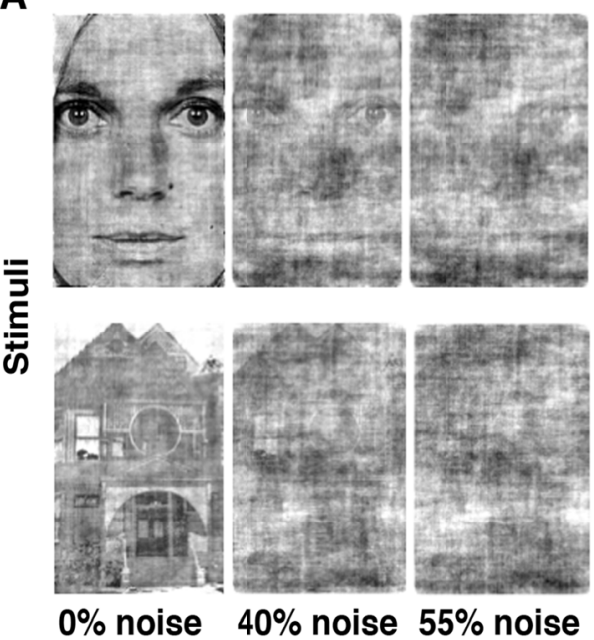

B

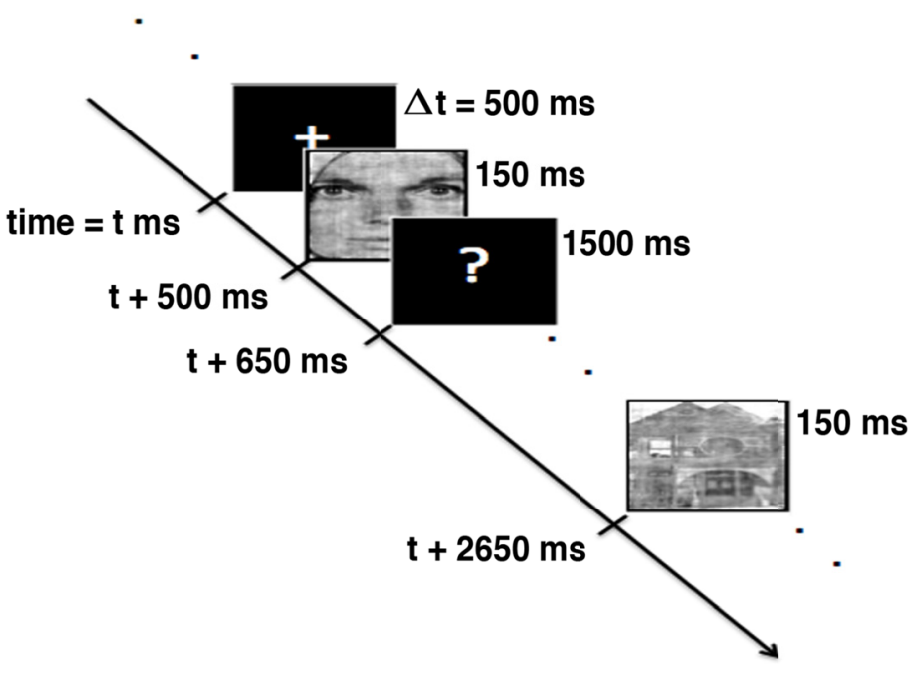

Fig. 1 

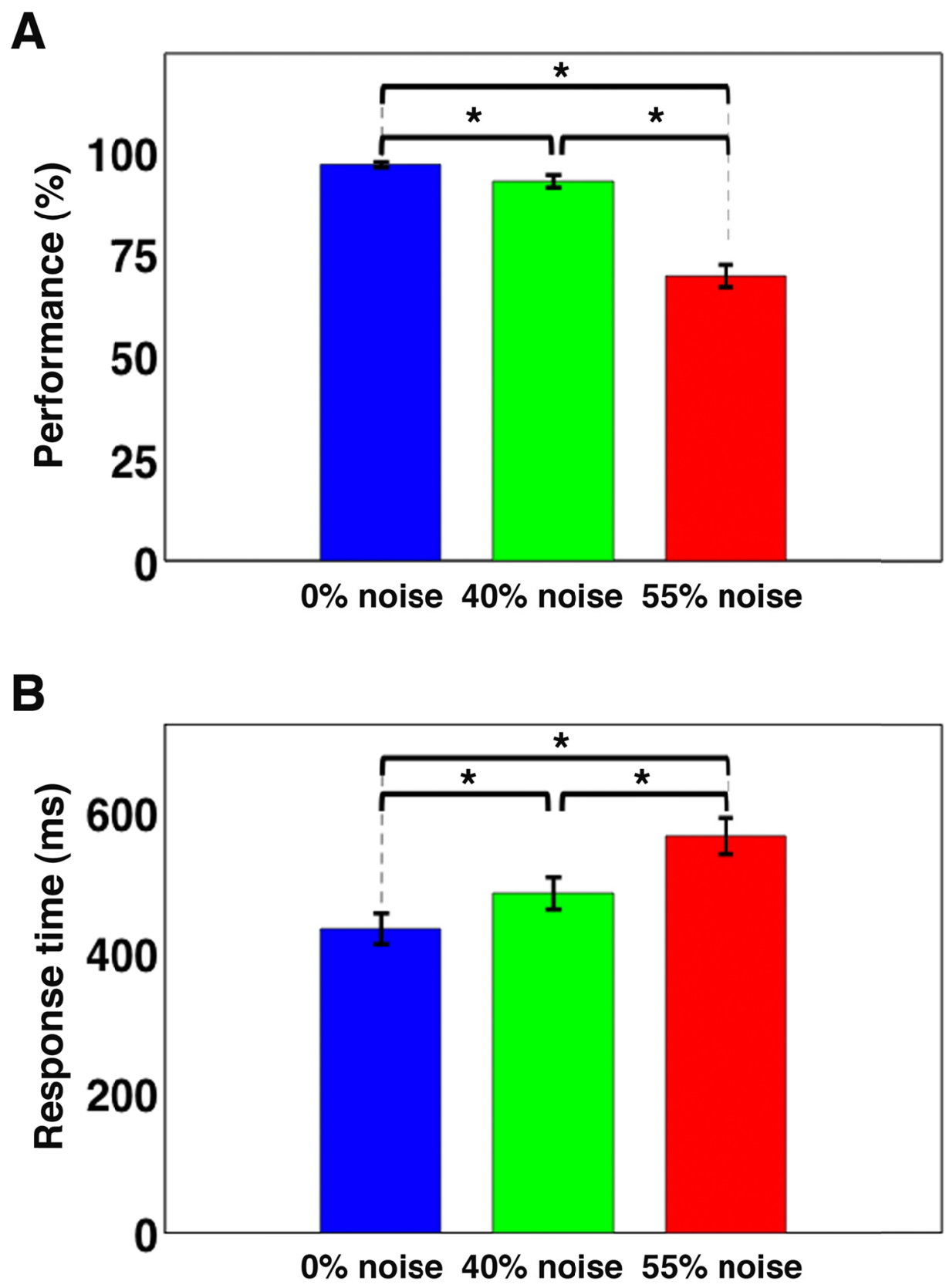

Fig. 2 

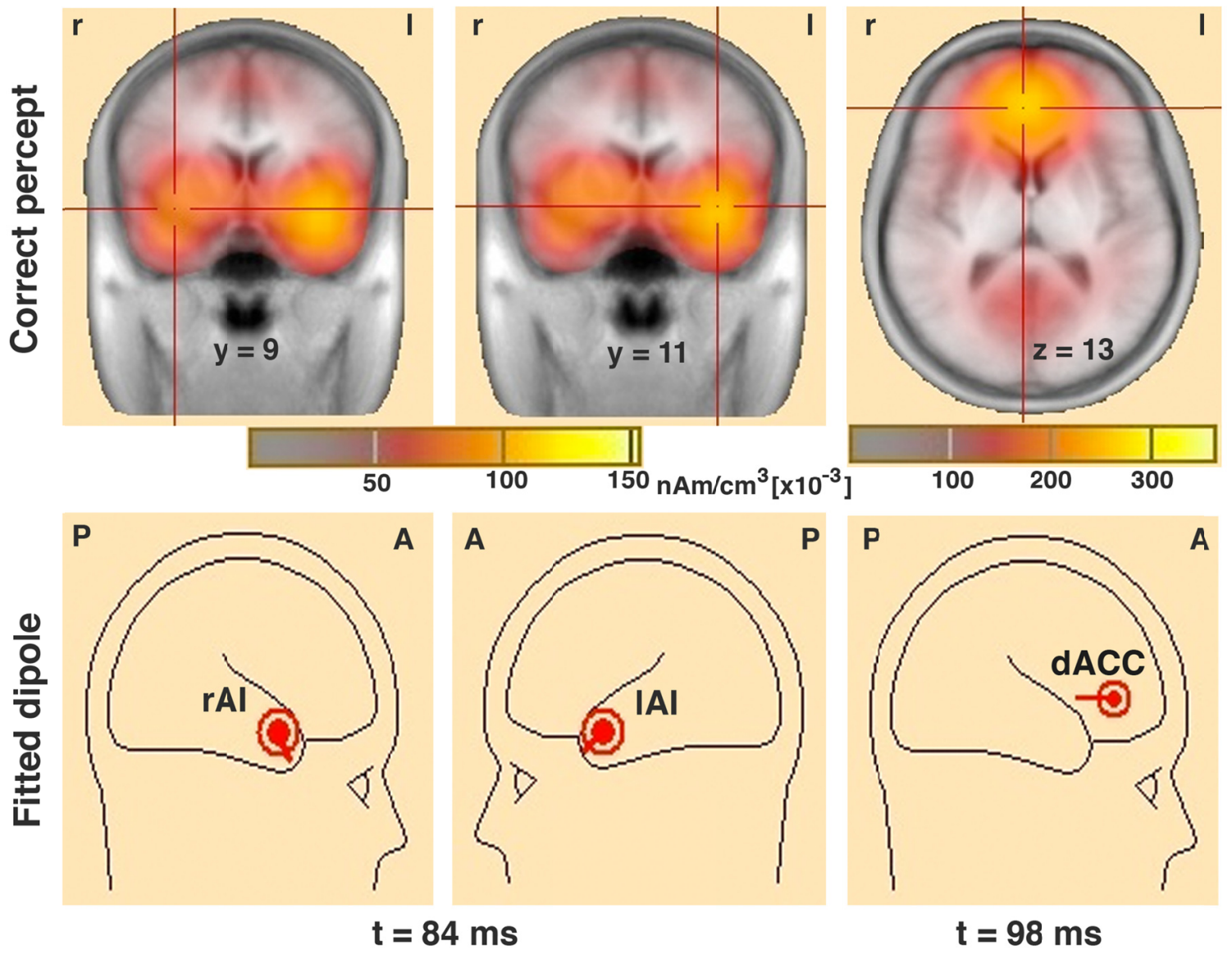

Fig. 3 

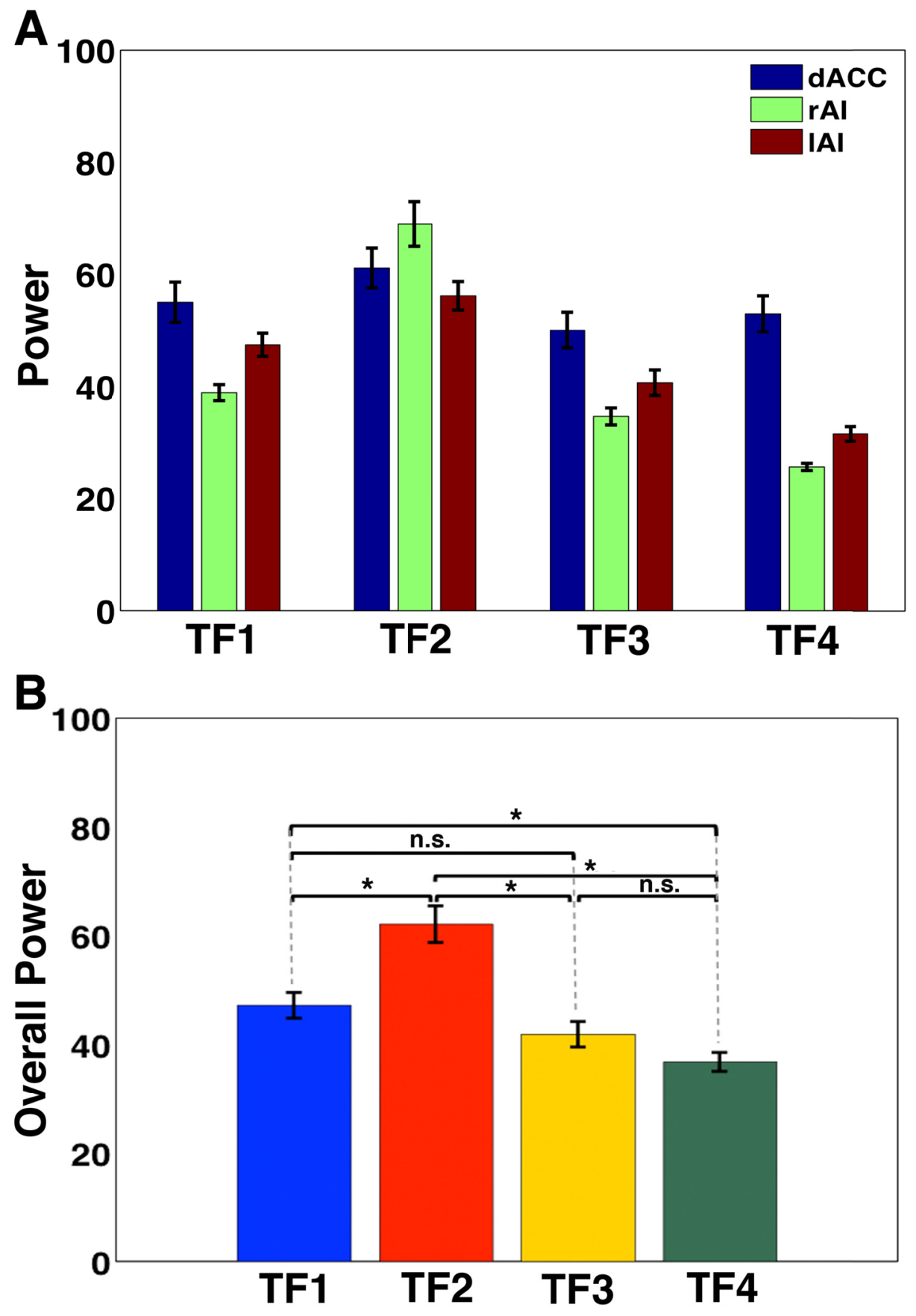

Fig. 4 

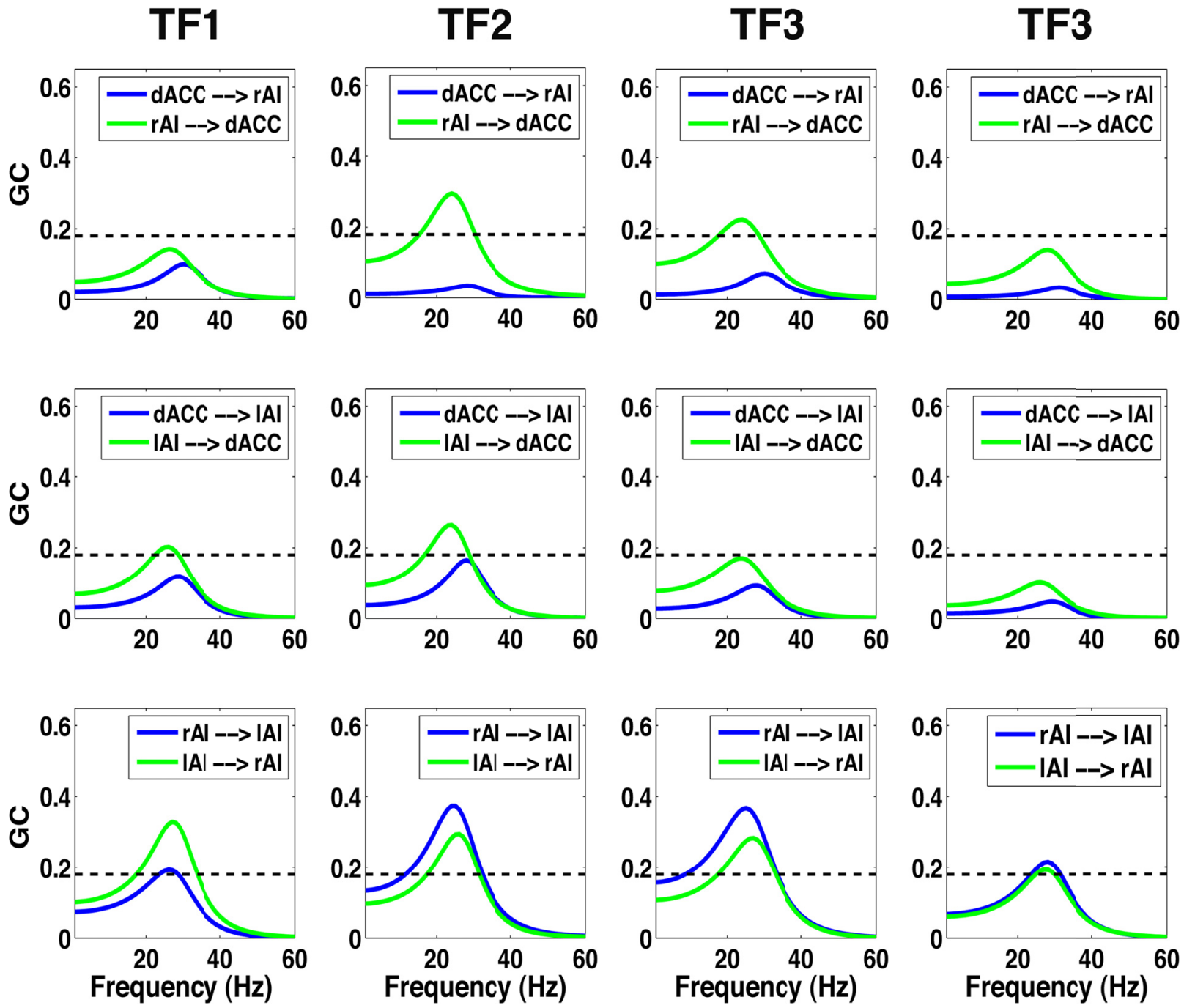

Fig. 5 

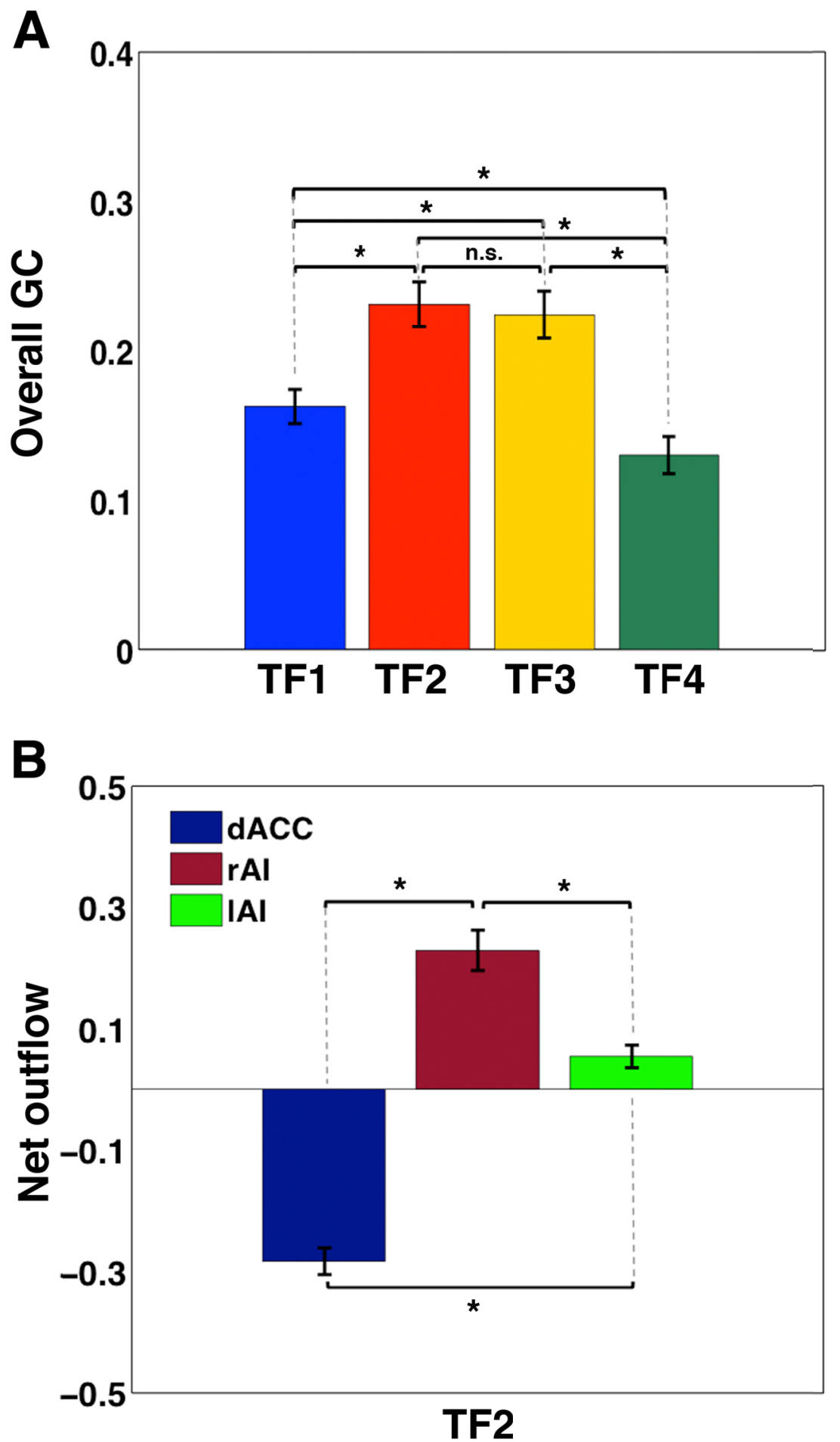

Fig. 6 


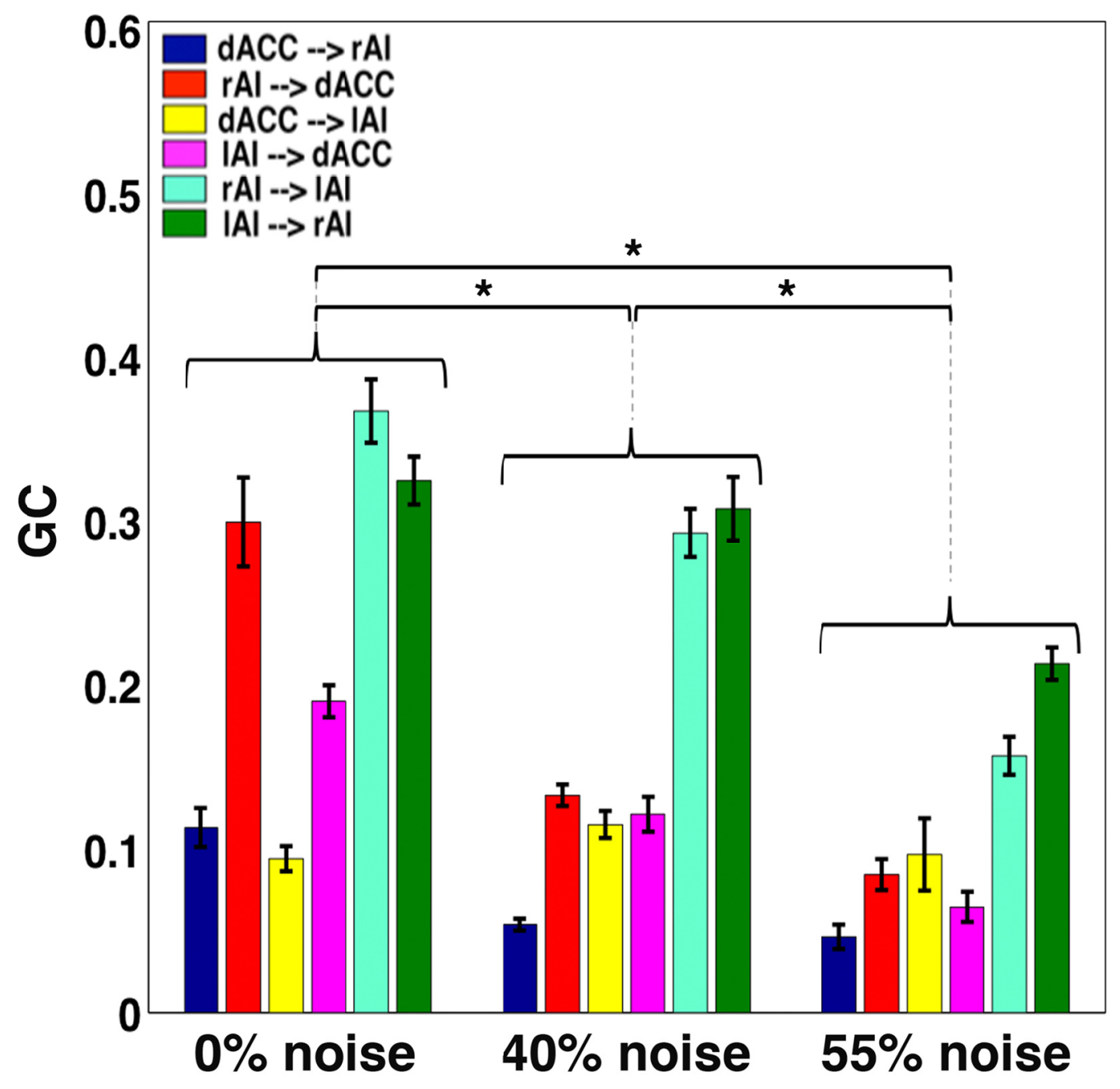

Fig. 7 
A

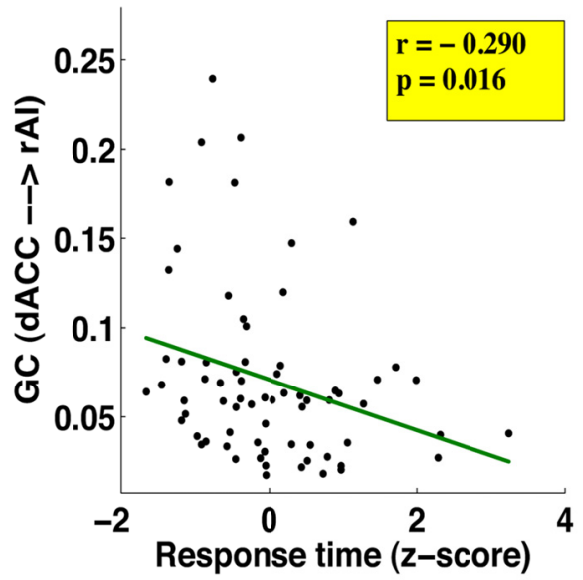

D

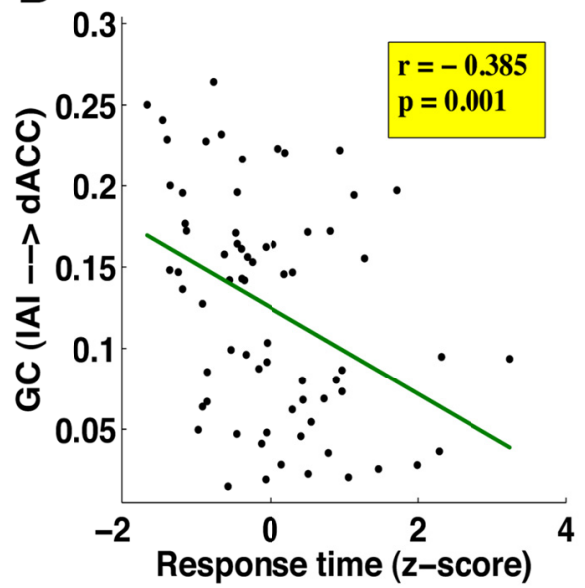

B

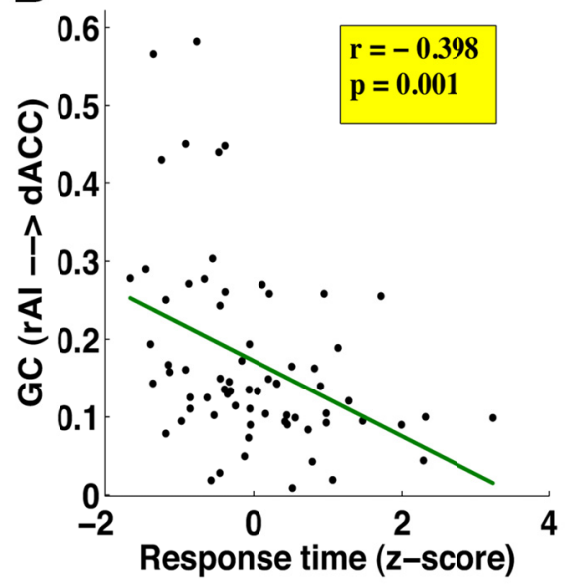

E

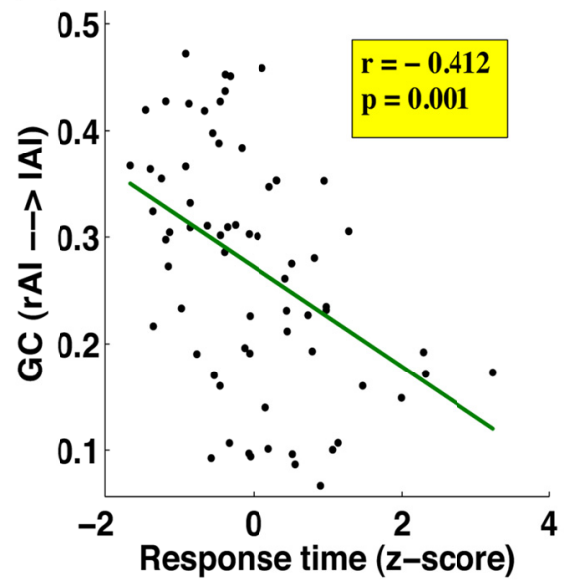

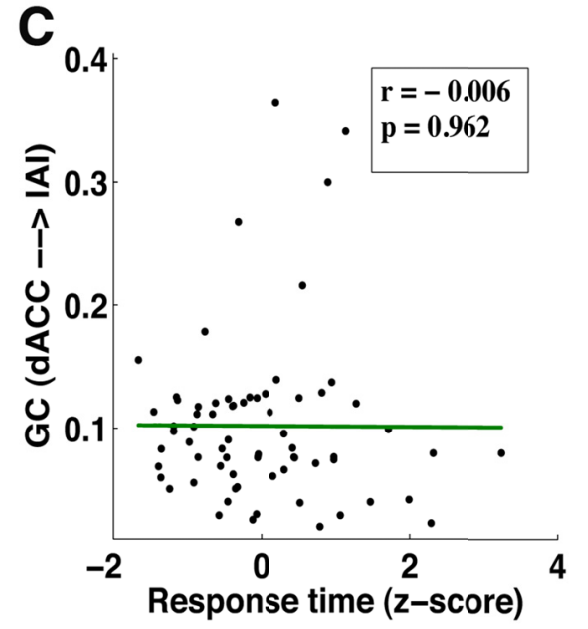

F

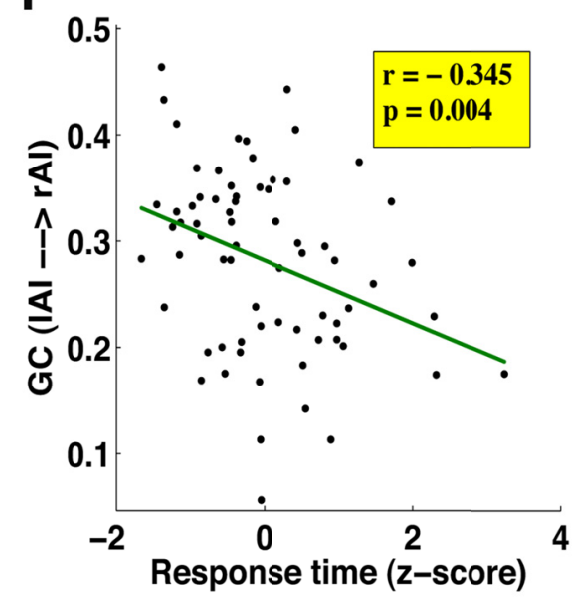

Fig. 8 


\begin{tabular}{|c|c|c|c|}
\hline Brain areas & $\begin{array}{c}\text { Talairach } \\
\text { coordinates } \\
(\mathbf{m m}) \\
\quad \mathbf{x}, \mathbf{y}, \mathbf{z}\end{array}$ & $\begin{array}{c}\text { Dipole orientations } \\
\qquad x, y, z\end{array}$ & $\begin{array}{c}\text { Dominant } \\
\text { activation period } \\
(\mathrm{ms})\end{array}$ \\
\hline $\begin{array}{l}\text { Right anterior insula } \\
\text { (rAI) }\end{array}$ & $35.0,9.0,-7.0$ & $0.9,0.3,-0.4$ & $78-142$ \\
\hline $\begin{array}{c}\text { Left anterior insula } \\
\text { (1AI) }\end{array}$ & $-33.0,11.0,-8.0$ & $-0.9,0.4,-0.3$ & $76-144$ \\
\hline $\begin{array}{l}\text { Dorsal anterior } \\
\text { cingulate cortex } \\
\text { (DACC) }\end{array}$ & $4.0,38.0,13.0$ & $0.1,1.0,0.0$ & $76-146$ \\
\hline
\end{tabular}

Table 1 